\title{
Parametric Optimization Design of Auto-unloading Mechanism Based on Adams
}

\author{
Yongliang Yuan \\ School of mechanical engineering, Dalian University of Technology, Dalian, China
}

\begin{abstract}
In order to improve the dynamic performance of a truck dump mechanism, the optimal parameter combination of the dump mechanism is studied. In combination with Adams' own modeling work, the dump mechanism is simplified and modeled and simulated. The objective function is optimized with the minimum lifting force, and the parameter combination of the best position relationship of the dump mechanism is obtained. The simulation results show that Adams can accurately predict the optimal parameter combination of mechanical devices, which have certain reference value for engineering design.
\end{abstract}

\section{Introduction}

Auto-unloading mechanism is a common and critical part of a dump truck and. Its performance will directly affect the overall performance of the dump truck. In order to improve the dynamic performance of dump trucks, many scholars have carried out relevant research on the lifting mechanisms and achieved some significant results ${ }^{[1-5]}$. According to the above research, it can be known that scholars' research mainly focused on the simplification, kinematics, and dynamics of the lifting mechanism. However, there are few researches on the optimization design of the lifting mechanism, and the influence of its optimal parameter combination has not been considered. Therefore, it is very important to carry out research on optimal parameter combination for parameters of dumping mechanism.

Adams is widely used dynamic simulation software of MSC, which has been widely used in machinery, aerospace, weapons and many other industries. In this paper, the parametric design of Adams is used to optimize the design of the dumping mechanism. The force of the hinge of the dumping mechanism is analyzed, and the minimum lift force is used as the objective function for optimization. The optimal position relationship of the dumping mechanism is obtained, which lays a foundation for the optimization design of the mechanism in the future.

\section{Parametric modeling}

\subsection{Establishment of dynamic model}

There are many ways to establish a dynamic model, which can be divided into two major categories. One is to use CAD software to establish, and the other is to use Adams' own modeling function. This paper considers that there are not very complicated components in the lifting mechanism. Therefore, the modeling method of this paper selects the latter.

In order to facilitate the optimization design research of the dump mechanism, the key points of the parts in the dump mechanism are parameterized to obtain the parametric model of the parameter dump mechanism.

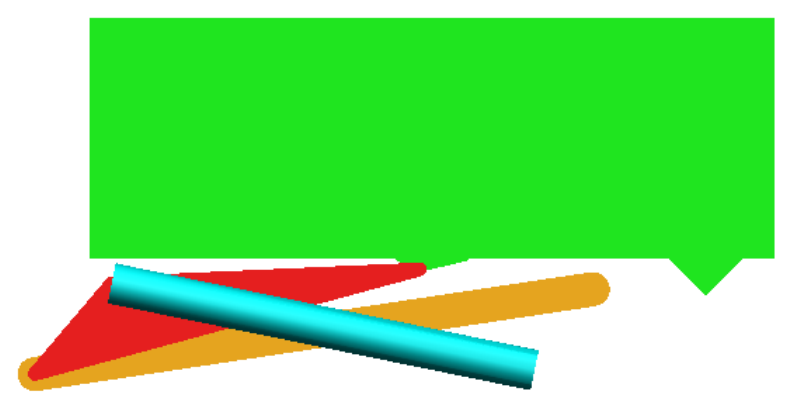

Figure 1. Parametric model of dumping mechanism.

The coordinate points in this paper are mainly the connection point between the cylinder and the earth, the connection point between the triangle arm and the carriage, and the connection point between the cylinder and the triangle arm. The variables are shown in Table 1.

Table 1. The parameterized variables.

\begin{tabular}{ccccc}
\hline & $\mathrm{X}$ & $\mathrm{Y}$ & Variable 1 & Variable 2 \\
\hline 1 & -3135 & 167 & $\mathrm{DV} \_1$ & $\mathrm{DV} \_2$ \\
2 & -2630 & 109 & $\mathrm{DV}$ _3 & DV_4 \\
3 & -2800 & -127 & DV_5 & DV_6 \\
\hline
\end{tabular}

\subsection{Constraints and Drivers}

According to the actual situation to add constraints, the constraints in the dumping mechanism includes: the hydraulic cylinder body and push rods add moving pairs, while the rest of the parts add rotating pairs. 
In order to facilitate the addition of the drive to the model, this paper ignores the influence of the oil inside the hydraulic cylinder and directly adds the speed of the end of the cylinder to the equivalent addition, and finally adds the model constraint ${ }^{[6-8]}$.

\section{Objective function and optimization variables}

Each of the optimization problem is determined by optimization variables and objective functions. Reasonable selection of optimization variables and objective functions is of great significance to the optimization results.

\subsection{Objective functions}

When the dumping device performs space movement, its overall performance is mainly affected by the lifting force. The lifting force tends to cause the carriage moving unsteadily. Conversely, it is easy to cause the discharge to be incomplete. Therefore, the paper chooses to optimize with the minimum lifting force as the objective function.

The lifting lift function selects the connection between the carriage and the triangular arm. To facilitate the selection of the objective function at a later stage, a measurement function is established, the direction of which is selected as the vertical direction.

\subsection{Design variables}

According to higher mechanics, the main factors affecting the objective function are the lengths of the rods that make up the dump mechanism. Therefore, the lengths of the three rods selected in this paper are used as optimization variables, and the specific variables can be determined by parameterized points. The design variables settings are shown in Table 2.

Table 2. Design variables settings.

\begin{tabular}{cccc}
\hline & Variable & Values & Range \\
\hline 1 & DV_1 & -3135 & {$[-3155,-3115]$} \\
2 & DV_2 & 167 & {$[157,177]$} \\
3 & DV_3 & -2630 & {$[-2650,-2610]$} \\
4 & DV_4 & 109 & {$[99,119]$} \\
5 & DV_5 & -2800 & {$[-3000,-2600]$} \\
6 & DV_6 & -127 & {$[-147,-107]$} \\
\hline
\end{tabular}

\section{Optimized design}

Adams is a multibody dynamics simulation software. In the simulation, assuming a lift angle of $46^{\circ}$ and a material weight of $5000 \mathrm{Kg}$, the lifting and unloading process is achieved by the relative movement of the hydraulic cylinder push rod and the triangular arm.
In order to simulate the dump truck unloading process more realistically, this paper uses the STEP function in Adams to describe the change of the load:

$5000+$ STEP( time, 0, 0, 15, -5000).

In this paper, the researcher chooses the Gear Stiff integrator (GSTIFF) and the integrator formulation (SI1), which takes into account the constraint derivatives when solving the equations of motion and monitors the integration error on the impulse of the Lagrange Multipliers in the system ${ }^{[9]}$. This work chooses the integration tolerance as 0.001 , the simulation time is $15 \mathrm{~s}$ and the step size is $0.02 \mathrm{~s}$.

After the first optimization, the values of various variables are shown in Table 3.

Table 3. The values of various variables.

\begin{tabular}{ccccc}
\hline & $\begin{array}{c}\text { Variabl } \\
\text { e }\end{array}$ & $\begin{array}{c}\text { Standard } \\
\text { value }\end{array}$ & $\begin{array}{c}\text { Optimal } \\
\text { value }\end{array}$ & Percentage \\
\hline 1 & DV_1 & -3135 & -3125 & 0 \\
2 & DV_2 & 167 & 159.63 & $-4.41 \%$ \\
3 & DV_3 & -2630 & -2629.5 & 0 \\
4 & DV_4 & 109 & 118 & $-8.25 \%$ \\
5 & DV_5 & -2800 & -2950 & $+5.36 \%$ \\
6 & DV_6 & -127 & -142 & $+11.8 \%$ \\
\hline
\end{tabular}

Lifting force curve is shown in Figure 2.

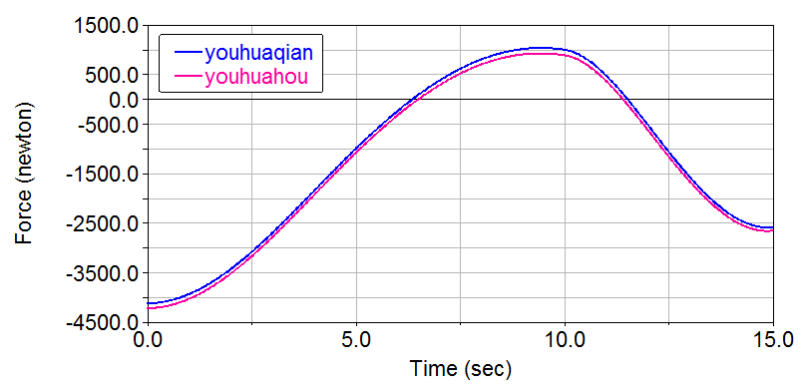

Figure 2. Lifting force curve.

From Figure 2, we can see that the maximum lifting force before optimization is $1050.8 \mathrm{~N}$, and the maximum value after optimization is $940.5 \mathrm{~N}$. The lifting capacity after optimization is about $1.35 \%$ smaller than before the optimization. The optimum values of the optimized variables is shown in Table 3.

Table 3. The values of optimized variables .

\begin{tabular}{cccc}
\hline & Variable & $\begin{array}{c}\text { Standard } \\
\text { value }\end{array}$ & Optimized values \\
\hline 1 & DV_1 & -3135 & -3145 \\
2 & DV_2 & 167 & 157 \\
3 & DV_3 & -2630 & -2629.5 \\
4 & DV_4 & 109 & 103.1 \\
5 & DV_5 & -2800 & -2950 \\
6 & DV_6 & -127 & -115.8
\end{tabular}

In this paper, the dynamic analysis is carried out for simulation based on the optimized results. The motion 
curve at each hinge is obtained. The speed curve of the carriage is shown in Figure 3.

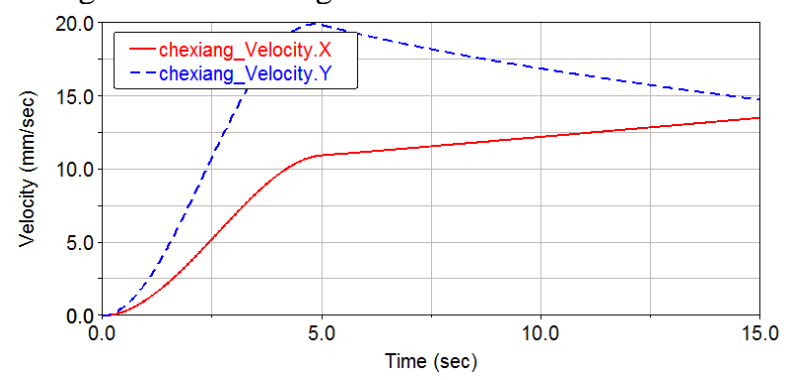

Figure 3. The speed curve of the carriage

As can be seen from Figure 3, when the dump mechanism is lifted, the lifting starts slowly due to a heavy object. As the weight of the unloaded car becomes lighter, the car lifts faster at the same speed. The slower speed of the car in the late period is mainly due to the fact that the simulation speed is too large, resulting in vibration of the car body and damage to the steel plate and other parts. Therefore, the speed in the later period should be relatively slow. The hinge force between the triangle arm and the carriage is shown in Figure 4.

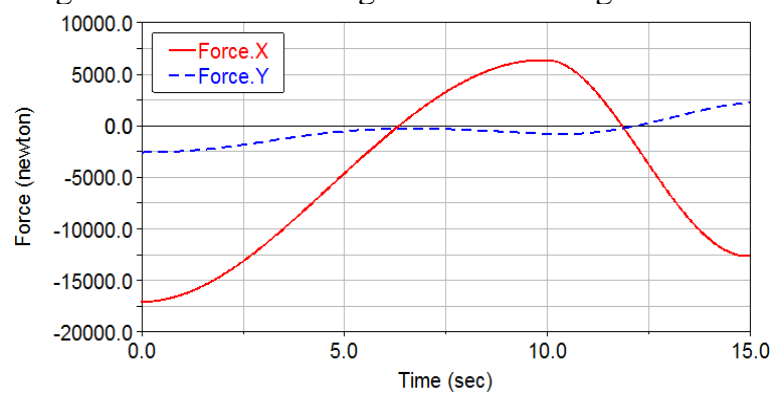

Figure 4. The hinge force between the triangle arm and the carriage.

By analyzing Figure 4, it can be seen that the influence of the hinge force between the triangle arm and the carriage in the $\mathrm{X}$ direction is obviously about the $\mathrm{Y}$ direction. The main reason is that the angle between the horizontal direction and the whole process from the beginning of the carriage unloading is less than 45 degrees. The force component of the cylinder push rod is caused by the large value in the $\mathrm{X}$ direction. Therefore, in order to improve the efficiency of the dump mechanism, there should be a certain angle between the hydraulic cylinder and the carriage in the design, not the parallel relationship.

\section{Conclusion}

This paper uses the parametric modeling function of Adams software to establish the model. The dynamic parametric model of the dump mechanism is completed, and a dynamic model is provided for the parameter optimization simulation analysis.

Through the parametric processing and optimization analysis of the coordinates of the interface of the dumping mechanism, the motion law and dynamics curve at the hinge are obtained. At the same time, the optimal parameter combination of the dump mechanism is obtained. The parametric optimization design of the dumping mechanism has certain practical significance for providing the dynamic performance of the dump truck, and provides a basis for future engineering design.

\section{References}

1. H.J. Yin. J. Mech. Des. 235 (2006).

2. X. Sun.J. Li. J. Const. Mach.. 407 (2009).

3. M.H. Vu, U.J. Na. IEEE T. Instrum. Meas. 6011 (2011).

4. K Nonami, R Yuasa, D Waterman. Auton. Robot. 183 (2005).

5. L.P. Chen, Y.L. Wang. More rigid body dynamics. Harbin engineering university press. (1995).

6. F. Qiu, Z. Yang, Y.L. Yuan. Packing Eng. 3614 (2015).

7. S.F. Wang, H.S. Li. Tractor. Farm. Trans. 364 (2009).

8. Y.L. Yuan, Z. Yang, S.H. Wang. Packing Eng. 35 11 (2014).

9. Y. Chen, Y. Sun, C. Chen. Mech. Mach. Theory. 98 (2016). 